\title{
OBSERVATIONS OF THE TWO-LEVEL STRUCTURE OF SUNSPOT MAGNETIC FIELDS
}

\author{
H. I. ABDUSSAMATOV \\ Pulkovo Observatory, U.S.S.R.
}

Inhomogeneity of magnetic field structure, 'granulation' in sunspot umbrae and fine structure of the Evershed motions lead to the conclusion that the sunspot umbra is composed of magnetic ropes (or plaits) with dimensions near the limit of resolution. Progress in the study of these ropes is closely connected with the possibility of obtaining extensive spectroscopic information about some selected regions on the solar disc. It is extremely interesting to obtain a picture of short-time-scale variations of the magnetic field strength and the radial velocity field in connection with the transfer of energy from the photosphere to the upper layers of the solar atmosphere (chromosphere, corona).

The four-camera isothermic spectrograph of the Pulkovo Observatory is a very suitable instrument for scanning an area amounting to $180^{\prime \prime} \times 45^{\prime \prime}$ per $53 \mathrm{~s}$. The scanning is performed by continuous moving of the solar image along the diurnal parallel and simultaneous automatic moving by steps of the four photographic plates after exposure. On each plate we take a series of spectra for neighboring positions of the slit on the disc. The number of spectral sections amounts to 24 .

When observing the structure of magnetic fields, a Wollaston prism is set behind the spectrograph slit and an achromatic $\lambda / 4$ plate - in front of the slit (Abdussamatov, 1970). In this case the observations are made in the 4th order only in three spectral regions $\left(\mathrm{H} \alpha, \lambda 6302 \AA\right.$ and $\left.\mathrm{D}_{1,2} \mathrm{Na}\right)$ with a linear dispersion of $3.7 \mathrm{~mm}$ per $\AA$ at $\mathrm{H} \alpha$.

A series of such plates characterizes the processes evolving in time and space (at least at two levels) in the solar atmosphere. The resolution is defined only by the seeing and image 'turbulence'. On June 22, 1968 the seeing conditions were good (resolution 2"). The leading and the following spots Group 241 (numeration from the Solnechnije Dannije) (Figure 1) were observed. We have taken 8 spectra on each plate at a distance of $5 . " 8$ from one another (the exposure time is $2 \mathrm{~s}$ ).

On Figure 2 ( $a, b$ and $c$ ) we see that the magnetic field, being somewhat diluted, penetrates into the chromosphere both over the leading and the following spots $\left(H_{\mathrm{chr}} \approx 0.5 H_{\mathrm{ph}}\right)$. If the geometrical difference of the two levels is $1500 \mathrm{~km}$ (White and Wilson, 1966) we have $\mathrm{d} H / \mathrm{d} h \approx 0.5-0.8 \mathrm{G} / \mathrm{km}$.

Investigation of the following spot shows that bright bridges can appear only as a consequence of fragmentation of magnetic fields of spots. Bright bridges are the inherent part of the structure of a spot umbra and not a formation lying over it (Abdussamatov, 1970).

Figure $2(\mathrm{a}, \mathrm{b}$ and $\mathrm{c})$ shows that the inhomogeneity of brightness in the spot is in good negative correlation with $H$. Dimensions of the structure elements nearly 


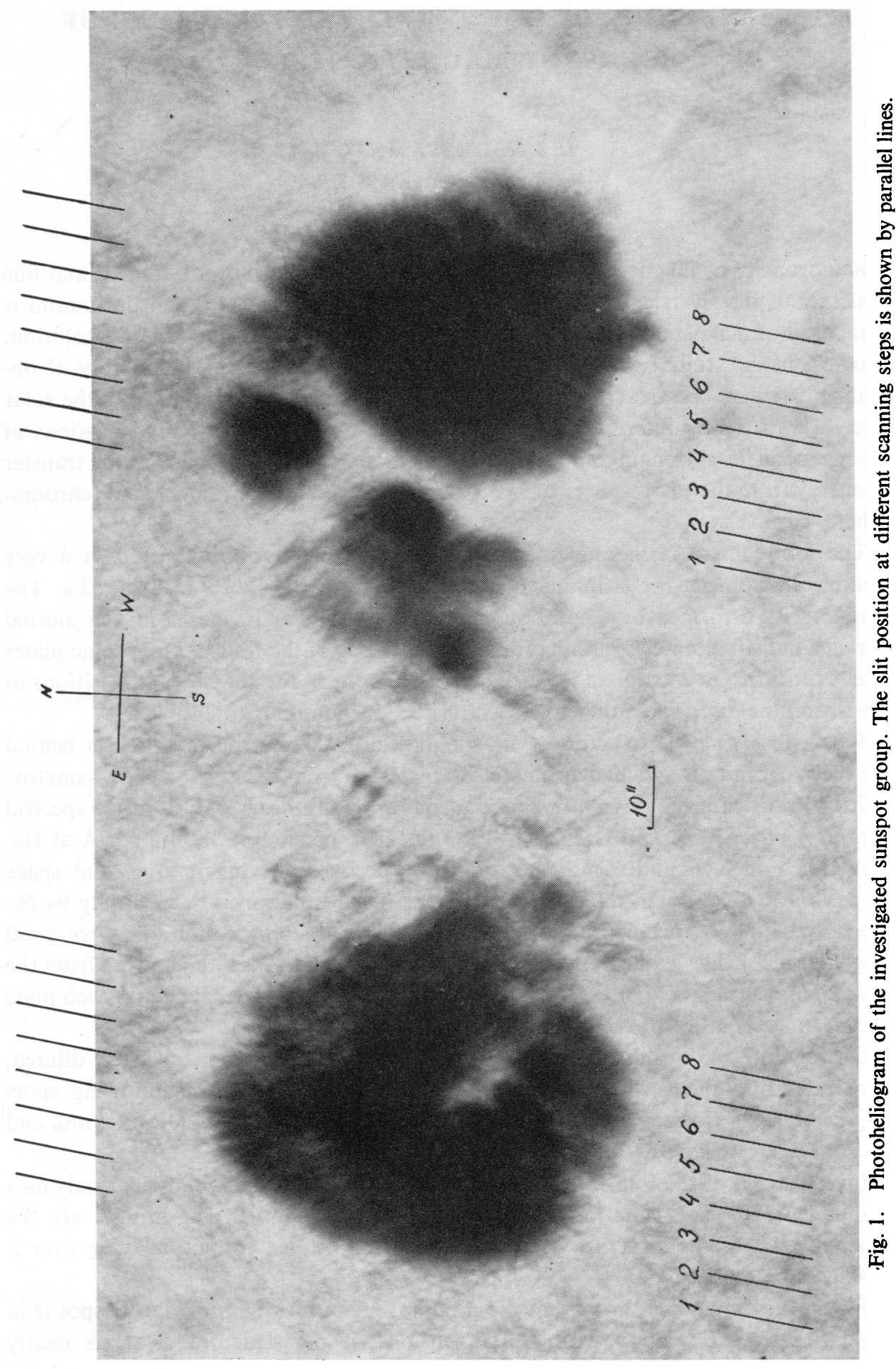




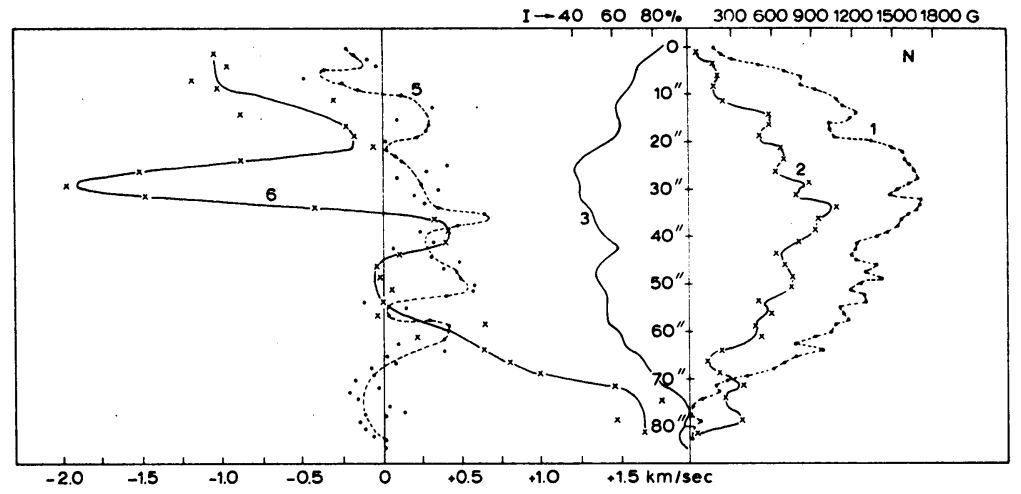

Fig. 2a.

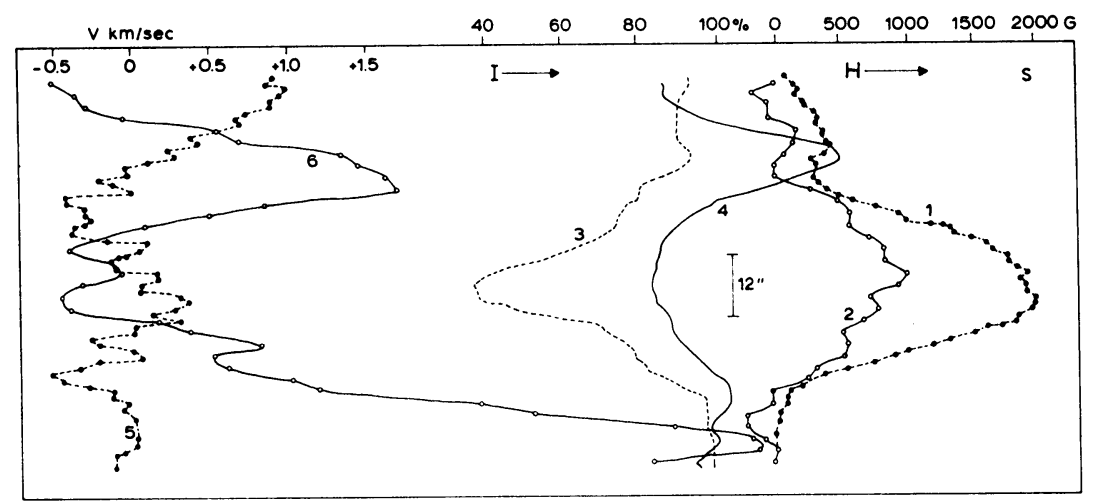

Fig. 2b.

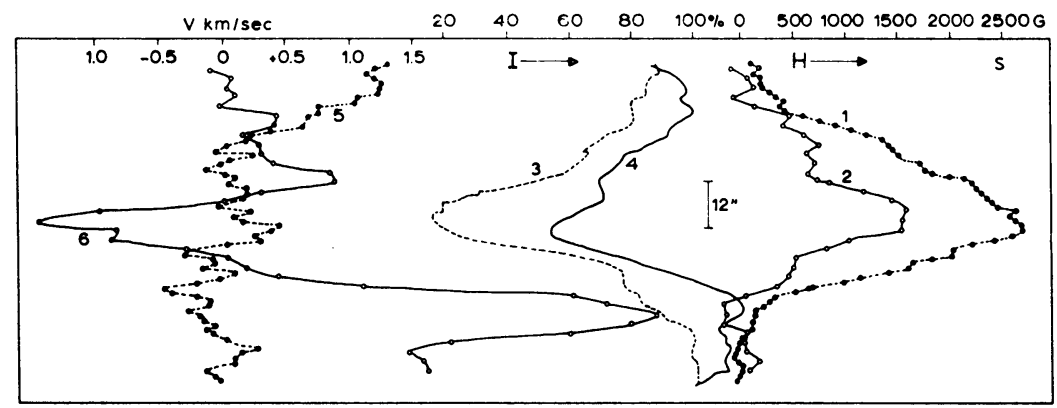

Fig. 2c.

Fig. 2a-c. (a) Photometric section of the east spot (follower) according to slit position 5 in Figure 1. (b) Photometric section of the west spot (leader) according to slit position 4. (c) Photometric section of the west spot according to slit position 5. 1-2 the distribution of the magnetic field strength on the photospheric and chromospheric levels respectively; 3-4 brightness distribution in the continuum and the center of the $\mathrm{H} \alpha$ line; 5-6 radial velocities at the photospheric and chromospheric levels. 


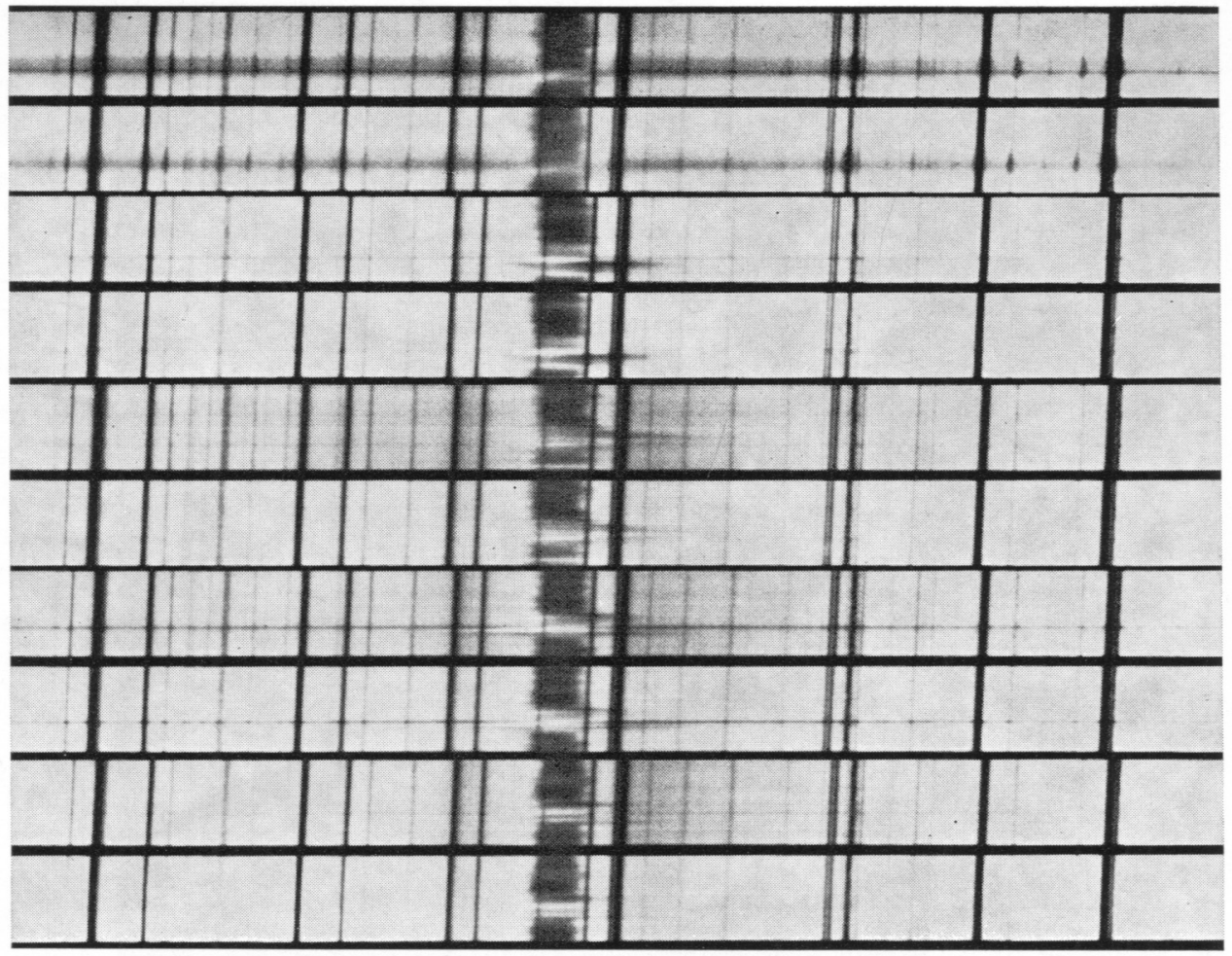

Fig. 3. Spectrograms of a solar flare obtained by the scanning method with the application of polarizational optics. ( $\mathrm{H} \alpha$ region, the length of the slit-80".)

correspond to that of the so-called magnetic 'knots'. It speaks in favor of the hypothesis (Abdussamatov and Krat, 1969) that the sunspot umbrae are composed of many magnetic ropes twisted.

The method described is very useful for investigations of flares; whereas the method of scanning permits us to obtain simultaneously (with good time resolution) not only a picture of the fine structure of magnetic fields and radial velocities of flares but also a series of characteristics derived from the profiles of spectral lines (Figure 3). By repeating the scans of a given area with short time intervals we can compare physical conditions in this region at the moment of the flare and after it.

\section{References}

Abdussamatov, H. I.: 1970, Astron. Zh. 47, 82.

Abdussamatov, H. I. and Krat, V. A.: 1969, Solar Phys. 9, 420.

White, O. R. and Wilson, P. R.: 1966, Astrophys. J. 146, 250. 